\title{
Non-gastrointestinal Bacillus cereus infections: an analysis of exotoxin production by strains isolated over a two-year period
}

\author{
PETER CB TURNBULL, JOHN M KRAMER \\ From the Food Hygiene Laboratory, Central Public Health Laboratory, Colindale Avenue, \\ London NW9 $5 H T$
}

SUMMARY Isolates of Bacillus cereus from 118 cases, and two maternity unit outbreaks, of nongastrointestinal infection were grouped on the basis of their estimated probable involvement in the infections from which they were isolated: (i) high probability-48 strains; (ii) intermediate - 16 strains; (iii) low -7 strains; (iv) very low ("irrelevant") 49 strains.

Rabbit skin test, haemolysin and phospholipase assays were used to determine exotoxin activities of strains within each group.

The results suggest a significant relation between the virulence of an isolate as reflected in the degree to which it appeared responsible for the signs and symptoms of an infection, and its toxigenicity in the skin test. This is attributed to the ability of $B$ cereus strains to synthesise, in varying degrees, a necrotic enterotoxin, possibly in conjunction with the primary haemolysin (cereolysin).

The cases analysed in this study support the contention that $B$ cereus, when isolated from an infection, may not be an inconsequential contaminant and should not be too readily dismissed as such.

The number of reports of infections caused by Bacillus cereus continues to increase..$^{1-4}$ They comprise a wide range of clinical conditions, often severe in nature and occasionally fatal. It has been proposed ${ }^{2}$ that an exotoxin, studied initially in relation to diarrhoeal-syndrome $B$ cereus food poisoning, ${ }^{56}$ is a principal determinant of virulence in nongastrointestinal $B$ cereus infections. This toxin has been partially purified and characterised, ${ }^{7}$ and shown to exhibit the properties of a true enterotoxin. ${ }^{8}$

Injected intradermally into rabbits the toxin produces increased vascular permeability and necrosis to a degree proportional to its concentration. However this test is not entirely specific for the enterotoxin: the $B$ cereus haemolysin, cereolysin, can also alter capillary permeability and cause similar dermal necrosis. In order to assess the contributory roles of these two exotoxins to the signs and symptoms of an infection, both the skin test and haemolytic activities of a clinical isolate must be determined.

Almost all $B$ cereus strains elaborate an extracellular phospholipase- $C$ (responsible for the diagnostic lecithinase reaction). Early beliefs that this metabolite might have a pathogenic role, possibly analogous to that of Clostridium perfringens $\alpha$-toxin (which also has lecithinase activity), are now in doubt. $^{3}$

The results of a previous analysis of the exotoxin activities of isolates from 21 human cases of $B$ cereus non-gastrointestinal infection and three cases of bovine mastitis indicated that only the vascular permeability reaction/necrotic toxin appeared to be related to the severity of the infection. ${ }^{2}$ The substantially larger number of isolates now being referred to this laboratory has enabled this more extensive investigation into the relation between exotoxin production and virulence to be undertaken. 


\section{Cases and methods}

\section{TYPES OF INFECTION FROM WHICH CULTURES} WERE RECEIVED

Isolates of $B$ cereus were received from a total of 136 cases encompassing a broad spectrum of infections: traumatic (postoperative or casualty) wounds and drain sites -74 cases (three deaths) including 10 described as necrotic or gangrenous; panophthalmitis-11 cases; bacteraemia/septicaemia-7 cases (excluding those placed within other categories such as wound or central nervous system infections with bacteraemia); abscesses and pustules -7 cases; peritonitis or ascites -7 cases; respiratory tract infections-6 cases (three deaths); burns-3 cases (one death); central nervous system infections - 3 cases (two deaths); "sticky" eyes in infants-3 cases; infected umbilici in infants-3 cases; ulcers -2 cases; cot death-1 case. Two maternity unit outbreaks ${ }^{910}$ accounted for a further 6 and 3 cases respectively: six of the affected infants had infected umbilical areas and three had upper respiratory tract infections.

\section{ASSESSMENT OF RELEVANCE}

Bacillus spp are commonly encountered in clinical laboratory cultures and tend to be regarded as environmental contaminants. It was therefore necessary to assess the relevance of the $B$ cereus isolates in the cases listed above. Specific details considered important to the assessment (Table 1) were sought from those clinicians and microbiologists directly involved in each case. Greater significance was attached to multiple isolations, particularly from blood cultures. Where $B$ cereus was not the only isolate recovered, attempts were also made to determine the proportions and types of other organisms present.

Fig. 1 illustrates how an assessment of relevance was made. At one extreme (upper left) a scanty $B$ cereus isolation in mixed culture from a patient showing no clinical evidence of infection was considered to be "irrelevant". At the opposite extreme (lower right) isolation of a heavy growth of the

Table 1 Criteria used to assess the relevance of Bacillus cereus to an infection

\footnotetext{
1 Isolation of $B$ cereus $\quad$ extent present $(+$ to ++++ ) -in pure/mixed culture

-on one/more than one occasion

2 Isolation of other organisms-identities and types (if known) -relative proportions (\%)

3 Nature and condition of infection site

4 Patient response (for example: pyrexia, toxic reaction)

5 Factors predisposing to infection

6 Relevant chemotherapy
}

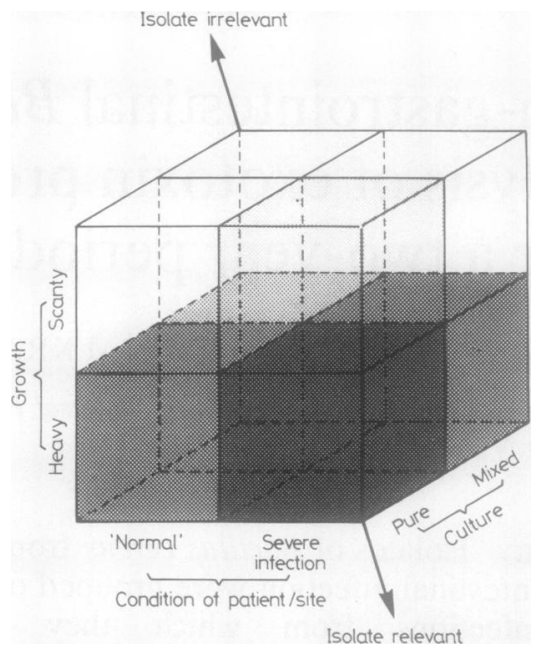

Fig. 1 Interrelation of principal factors used to assess the relevance of Bacillus cereus to an infection.

organism in pure culture from an obviously infected site, with the patient in a toxic state, was regarded as being "relevant".

Many isolates, as expected, fell between these two extremes. So, for the purposes of analysing the importance of exotoxin production to the clinicalco condition apparent, strains were placed into one of $\rightleftharpoons$ four categories of probable relevance.

Sufficient information was available on 105 of the 136 cases to permit classification in this way. Serological typing and consistent toxin profiles indicated that the two outbreaks of non-gastrointestinal $B$ cereus infection could be.represented by just two strains.

A further 21 cases of human infection previously reported $^{2}$ were re-examined under the new criteria of assessment: the information on 13 of these was judged adequate to permit their inclusion in this study. The analysis was therefore carried out on a total of 120 strains.

\section{ASSAYS OF SKIN TEST, HAEMOLYTIC AND} PHOSPHOLIPOLYTIC ACTIVITIES

The methods used were essentially as described previously. ${ }^{27}$ For toxin production, $18 \mathrm{~h}$ nutrient broth cultures of the test strains were used to inoculate $10 \mathrm{ml}$ volumes of brain heart infusion broth (Difco) $+0.1 \% \mathrm{wt} / \mathrm{vol}$ glucose in $250 \mathrm{ml}$ Erlenmeyer flasks. These were incubated with shaking ( $200 \mathrm{cycles} / \mathrm{min})$ for $6-7 \mathrm{~h}$ at $36^{\circ} \mathrm{C}, \mathrm{pH}$ control being by addition of $0.1 \mathrm{ml} 1 \mathrm{~N} \mathrm{NaOH}$ to each culture at $3-3.5 \mathrm{~h}$ and again at $4-4 \cdot 5 \mathrm{~h}$. Bacterial cells were removed by centrifugation and filtration through $0.45 \mu \mathrm{m}$ membrane filters (Millipore Ltd, London). 
For the rabbit skin test assay, $0.05 \mathrm{ml}$ of each cell-free culture filtrate (CCF) was injected intradermally in duplicate into the depilated backs of two adult rabbits. At $3 \mathrm{~h}$ postinoculation, $4 \mathrm{ml}$ of $2 \%$ $\mathrm{wt} / \mathrm{vol}$ Evans Blue (Sigma, London) was injected intravenously; measurements of zones of blueing and necrosis were made after a further hour. Necrotic activity, when present, was rechecked at $24 \mathrm{~h}$. In previous studies, ${ }^{237}$ skin test readings were placed into five categories ranging from very weak (category 1 ) to very strong (category 5) toxin production. However, it has since proved more practical to use just three groups, designated "weak" (formerly categories 1 and 2), "intermediate" (formerly category 3 ) and "strong" (formerly categories 4 and 5).

Haemolysin titrations were carried out in WHO plates using twofold serial dilutions of CCF incubated for $2 \mathrm{~h}$ at $36^{\circ} \mathrm{C}$ with $0.5 \% \mathrm{vol} / \mathrm{vol}$ washed rabbit erythrocytes suspended in $0.01 \mathrm{M}$ phosphate buffered saline. Test strains were divided into four groups on the basis of their reciprocal haemolysin titres: $<10,20-80,160-640$, and $>640$.

Phospholipase activities were determined as the mean diameters of zones of opacity produced (after $20 \mathrm{~h}$ at $36^{\circ} \mathrm{C}$ ) in a $10 \% \mathrm{vol} / \mathrm{vol}$ egg-yolk emulsion agar around duplicate $5 \mathrm{~mm}$ wells containing $50 \mu \mathrm{l}$ CCF. Strains were allocated to one of three categories according to their phospholipolytic activity: $<10 \mathrm{~mm}, 10-20 \mathrm{~mm}$, and $>20 \mathrm{~mm}$.

\section{Results}

\section{RELEVANCE OF ISOLATES}

The 120 strains included in this study were grouped according to their probable relevance to the infections from which they were isolated, as follows: (i) high probability - 48 strains; (ii) intermediate16 strains; (iii) low -7 strains; (iv) very low (of no clinical significance) - 49 strains.

With reference to the 10 patients who died it was considered that $B$ cereus infection was a contributory factor in the deaths of seven (intermediate probability of relevance in six; low probability in one), and that in a further two cases (including the cot death) the organism probably did not have any role. The significance of the $B$ cereus isolation in the remaining case could not be assessed.

\section{EXOTOXIN PRODUCTION VERSUS RELEVANCE}

The histograms (Figs. 2, 3 and 4) show the relations found between the rabbit skin test, haemolytic and phospholipolytic activities of the strains and the estimated relevance to their respective infections. For further comparison, the results of tests on environmental isolates accumulated over some years were also included. These strains were isolated from dairy products, meat and poultry, hospital environments, animal feedstuffs and other miscellaneous sources.

In Fig. 2 a marked bias is apparent towards strong skin test activity among the strains assessed as having some degree of clinical relevance, particularly those in the "high" or "intermediate" probability categories. In contrast, strains assessed as being of "very low" relevance show a bias towards weak skin test activity. Furthermore, an approximately normal distribution of toxigenicity was found among the 68 environmental isolates.

In Fig. 3, haemolysin production by the same clinical isolates is analysed. Haemolysin titrations were only carried out on 27 of the environmental isolates. As with the skin test activity, there is a notable bias towards high haemolysin titres among those isolates considered to have some degree of clinical relevance, although the contrast between the "relevant" and the "irrelevant" strains is not as marked as in Fig. 2. Nonetheless, haemolysin production by the "irrelevant" strains lacked any bias towards high titres and had a distribution comparable with that of the environmental isolates.

From a similar analysis of phospholipase production (Fig. 4) a bias towards higher enzyme activity

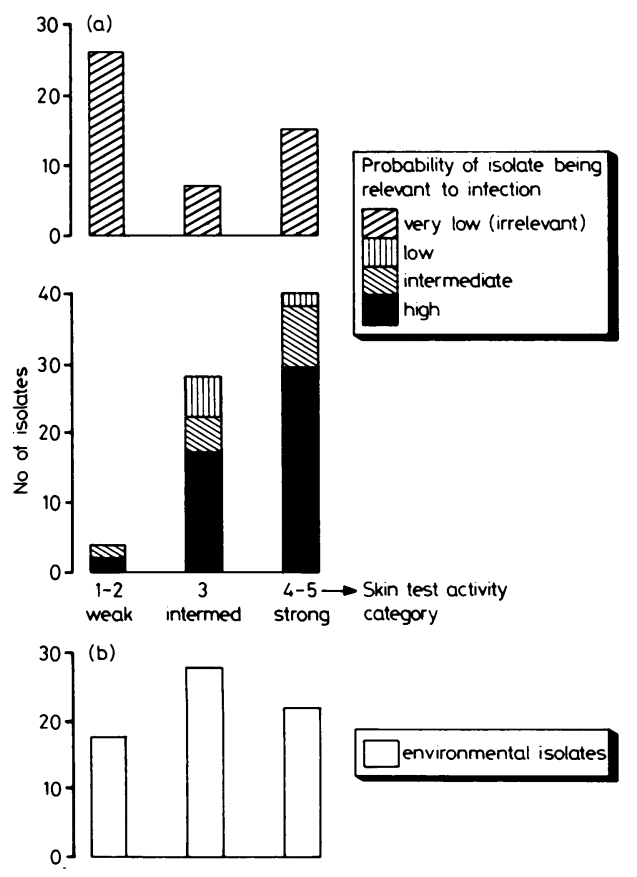

Fig. 2 Skin test toxigenicity of (a) 120 clinical isolates of Bacillus cereus and (b) 68 environmental isolates. 


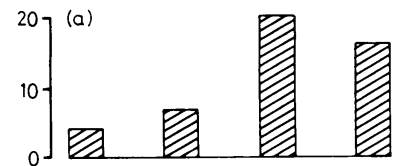

Probability of isolate being relevant to intection Vuery low (irrelevant) IIIII) low
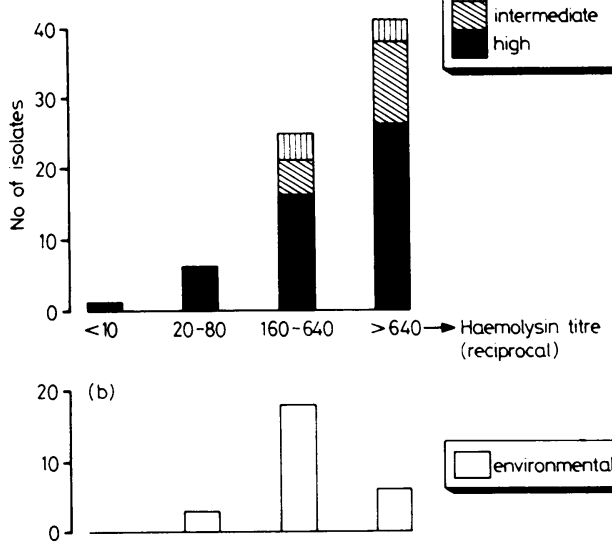

environmental isolates

Fig. 3 Haemolysin production by (a) 120 clinical isolates of Bacillus cereus and (b) 27 environmental isolates.

was shown equally by strains of $B$ cereus from all sources.

Without supplying absolute proof, the results presented in Fig. 2 indicate a correlation between the

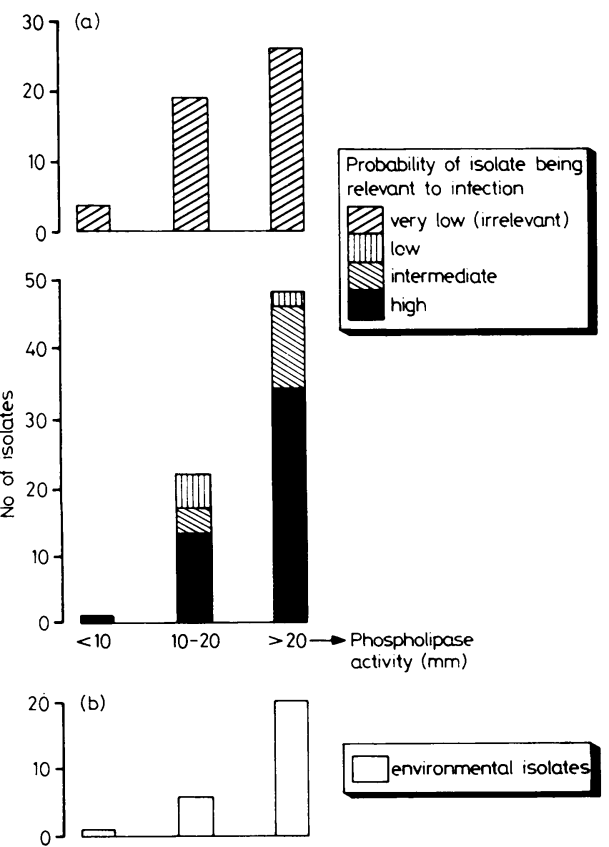

Fig. 4 Phospholipase production by (a) 120 clinical isolates of Bacillus cereus and (b) 27 environmental isolates. degree of reaction obtained with the rabbit skin test and the reported manifestation of symptoms.

Support for this conclusion seems to lie in the contrasting absence of any apparent association between phospholipase production and assessed relevance of a strain to its associated infection (Fig. 4); there is no obvious difference between strains deemed to have caused or contributed to symptoms, those considered irrelevant and the environmental strains.

Conclusions with respect to haemolysin production (Fig. 3) are less easy to make. A bias towards high titres is an apparent characteristic of those strains judged relevant to some degree. This bias is essentially absent among the "irrelevant" strains. However, a contrasting bias towards low skin test activity by the latter was not apparent either, and distribution curves for the "irrelevant" and environmental strains were both similar. Possible interpretations of the haemolysin results are discussed below.

\section{ANTIMICROBIAL SENSITIVITIES}

The results of in vitro antimicrobial sensitivity tests, carried out in all instances by disc diffusion, were supplied with 68 of the clinical isolates. These data are summarised in Table 2 .

\section{Discussion}

The cases analysed in this study in which $B$ cereus was considered either contributory or causative, substantially enhances the increasing evidence that this bacterium should be viewed more seriously as a potential pathogen and not be too readily dismissed as a saprophyte.

Underlying disease or debilitation did not feature greatly in the histories obtained on these cases: seven patients were under treatment for malignancies, one was on steroid therapy and one a diabetic. Apart from two premature infants, the remainder of

Table 2 In vitro antimicrobial sensitivities of 68 strains of Bacillus cereus isolated from non-gastrointestinal infections

\begin{tabular}{lcrrr}
\hline Antimicrobial & \multicolumn{5}{c}{ No of strains } \\
\cline { 2 - 5 } & \multicolumn{5}{c}{ Sensitive $\begin{array}{l}\text { Intermed- } \\
\text { iate }\end{array}$} & Resistant \\
\hline Cefuroxime & $30 \mu \mathrm{g}$ & 0 & 1 & 24 \\
Cephaloridine & $25 \mu \mathrm{g}$ & 1 & 37 & 2 \\
Chloramphenicol & $10 \mu \mathrm{g}$ & 27 & 0 & 0 \\
Erythromycin & $5 \mu \mathrm{g}$ & 59 & 7 & 0 \\
Gentamicin & $10 \mu \mathrm{g}$ & 67 & 1 & 0 \\
Methicillin & $10 \mu \mathrm{g}$ & 8 & 1 & 17 \\
Penicillin G & $1 \mathrm{unit}$ & 1 & 4 & 61 \\
Tetracycline & $10 \mu \mathrm{g}$ & 49 & 7 & 9 \\
Trimethoprim & $2.5 \mu \mathrm{g}$ & 0 & 0 & 26 \\
Sulphonamide & $50 \mu \mathrm{g}$ & 23 & 1 & 2 \\
\hline
\end{tabular}


the patients appeared to have been in good health prior to the event (accident, burns, surgery etc.) which led to the $B$ cereus infection.

As a clinical entity, the proportion of cases of panophthalmitis stands out noticeably. The importance of $B$ cereus as a cause of panophthalmitis has been discussed elsewhere. ${ }^{11}$

The seven deaths associated with $B$ cereus infections included four cases of respiratory tract involvement, two cases (one a premature neonate) of central nervous system involvement and one (also a premature neonate) involving both the respiratory tract and central nervous system. $B$ cereus is known to produce a "lethal toxin" the nature of which is becoming clearer, ${ }^{3}$ and the lethal potential of this organism has been observed in both naturally ${ }^{12}$ and experimentally ${ }^{1314}$ infected animals.

Age and predisposition to infection did not appear related. In the two maternity unit outbreaks, the infections in the first ${ }^{9}$ were mild; in the second, ${ }^{10}$ only three of 28 infants from which the common serotype was isolated exhibited clinical signs, and from these Staphylococcus aureus was also isolated. Of the remaining cases in which the $B$ cereus isolations were considered relevant, a further two were infants and one was recorded as elderly.

With reference to possible modes of $B$ cereus pathogenesis, the results support previous contentions $\mathrm{s}^{23714}$ that there is a significant relation between the skin test toxigenicity of a strain and the extent of clinical manifestation of the infection.

The nature of the skin test reaction has been reviewed extensively. ${ }^{3}$ The response in the skin of rabbits or guinea pigs to intradermal injection of crude $B$ cereus culture filtrates is attributable to a combination of two distinct factors: (i) a partially characterised necrotic enterotoxin of a molecular weight of approximately 50000 daltons and (ii) the primary $B$ cereus haemolysin, cereolysin. Their individual roles can, as yet, only be assessed indirectly by testing separately the haemolytic activity of the culture filtrate as was done here. In its purified form the enterotoxin has little or no inherent in vitro haemolytic activity,' though this is something of a paradox since it possesses strongly necrotic and cytotoxic properties. Furthermore, certain strains at least, are known to produce a secondary (minor) haemolysin. For these reasons in vitro haemolysis tests as used here are not entirely satisfactory and alternative immunological assays are awaited.

The finding of a similar, though less marked, correlation between the haemolytic activity of an isolate and severity of infection suggests that haemolysin production is also involved in $B$ cereus pathogenicity. However some qualification is necessary since cereolysin activity has been shown to be neutralised in vitro by normal serum. ${ }^{3}$ The manner and extent, if any, by which this occurs in vivo is not yet known.

The results also indicate that phospholipase- $\mathrm{C}$ is probably not of direct pathological significance per $s e$ in $B$ cereus infections. There is some evidence ${ }^{3}$ to suggest that phospholipase- $C$ might play an auxiliary pathogenic role by breaking-down host-cell membrane phospholipids exposed by the action of other enzymes or toxins. The contribution of "secondary virulence factors" would not necessarily be apparent in a study of this kind.

An incidental observation made during this investigation was the distribution of serotypes among the $B$ cereus isolates: $13.5 \%$ of strains were serotype 20 , a further $25.6 \%$ fell into 18 different serogroups, and $60.9 \%$ were not typable. The reason for the prevalence of type 20 among human isolates is not clear, but a similar phenomenon has been noted in investigations of $B$ cereus emetic-syndrome food poisoning, where $70 \%$ of isolates were type $1,{ }^{15}$ and $B$ cereus -induced bovine mastitis, where strains of serotype 12 were predominant. $^{12}$ The unusual degree of heat resistance exhibited by type 1 spores $^{16}$ may account for the frequent association of this serotype with food poisoning, but no analogous explanation has yet been found for the disproportionate representation of types 20 and 12 in human and veterinary isolations.

The authors warmly acknowledge their debt to the many clinicians and microbiologists who supplied the cultures and isolation histories without which this study would not have been possible. We also greatly appreciate the invaluable help given by $\mathrm{Dr}$ RJ Gilbert, Dr D Roberts, Mrs C Putman and Mr EA Leeson (Food Hygiene Laboratory), Mr P Gerson and staff (Animal Department) and Mr JR Gibson and staff (Department of Medical Illustration), Central Public Health Laboratory.

\section{References}

1 Tuazon CU, Murray HW, Levy C, et al. Serious infections from Bacillus sp. JAMA 1979;241:1137-40.

${ }^{2}$ Turnbull PCB, Jørgensen K, Kramer JM, et al. Severe clinical conditions associated with Bacillus cereus and the apparent involvement of exotoxins. J Clin Pathol 1979;32:289-93.

${ }^{3}$ Turnbull PCB. Bacillus cereus toxins. Pharmacol Ther 1981;13:453-505.

4 Shamsuddin D, Tuazon CU, Levy C, Curtin J. Bacillus cereus panophthalmitis: source of the organism. Rev Infect Dis 1982;4:97-103.

s Spira WM, Goepfert JM. Biological characteristics of an enterotoxin produced by Bacillus cereus. Can J Microbiol 1975;21:1236-46.

- Turnbull PCB. Studies on the production of enterotoxins by Bacillus cereus. J Clin Pathol 1976;29:941-8.

7 Turnbull PCB, Kramer JM, Jørgensen K, et al. Properties and production characteristics of vomiting, diarrheal, and nec- 
rotizing toxins of Bacillus cereus. Am J Clin Nutr 1979;32:219-28.

- Bonventre PF, Lincoln RE, Lamanna C. Status of bacterial toxins and their nomenclature: need for discipline and clarity of expression. Bact Rev 1967;31:95-109.

9 World Health Organization. Bacillus cereus in a maternity unit. Weekly Epidemiological Record 1981;12:93.

${ }^{10}$ Birch BR, Perera BS, Hyde WA, et al. Bacillus cereus crossinfection in a maternity-unit. J Hosp Infect 1981;2:349-54.

"O'Day DM, Smith RS, Gregg CR, et al. The problem of Bacillus species infection with special emphasis on the virulence of Bacillus cereus. Ophthalmology (Rochester) 1981;88:833-8.

12 Jones TO, Turnbull PCB. Bovine mastitis caused by Bacillus cereus. Vet Rec 1981;108:272-4.

13 Balacescu C. Über einen von Bacillus cereus synthetitisierten "lethal factor". Zentralbl Bakteriol [B] 1975;161:178-87.

14 Turnbull PCB, Nottingham JF, Ghosh AC. A severe necrotic enterotoxin produced by certain food, food poisoning and other clinical isolates of Bacillus cereus. Br J Exp Pathol 1977;58:273-80.

${ }^{15}$ Kramer JM, Turnbull PCB, Munshi G, Gilbert RJ. Identification and characterization of Bacillus cereus and other Bacillus species associated with foods and food poisoning. In: Corry JEL, Roberts D, Skinner FA, eds. Methods for the isolation and identification of food poisoning organisms. Soc Appl Bacteriol Technical Series No 17. London: Academic Press, 1982: 261-86.

${ }^{16}$ Parry JM, Gilbert RJ. Studies on the heat resistance of Bacillus cereus spores and growth of the organism in boiled rice. J Hyg (Lond) 1980;84:77-82.

Requests for reprints to: JM Kramer, Food Hygiene Laboratory, Central Public Health Laboratory, 175 Colindale Avenue, London NW9 5HT, England. 